\title{
A Defense of the Paraconsistent Approach to Quantum Superpositions (Reply to Arenhart and Krause)*
}

Una defensa del enfoque paraconsistente de las superposiciones cuánticas (respuesta a Arenhart y Krause)

Christian de Ronde

\begin{abstract}
In da Costa and de Ronde (2013) we discussed the possibility of considering quantum superpositions in terms of a paraconsistent approach. We argued that, even though most interpretations of quantum mechanics attempt to escape contradictions, there are many reasons that indicate it could be worthwhile to engage in a research of this kind. Recently, Arenhart and Krause (2016) have raised several arguments against this Paraconsistent Approach to Quantum Superpositions (PAQS). In this paper I attempt to answer the main questions and obstacles presented by them. I will argue, firstly, that the obstacles addressed by Arenhart and Krause are based on a specific stance grounded on an actualist metaphysics-which implicitly presupposes classical logic. Secondly, that there are many interpretations that investigate the possibility of developing a different mode of existence to that of actuality for which the PAQS might be regarded as a valuable prospect. Finally, we provide a set of specific answers to the main problems raised by Arenhart and Krause in order to clarify the original perspective introduced by the PAQS.
\end{abstract}

Keywords: quantum superpositions - paraconsistency - measurement problem

\section{Resumen}

En da Costa y de Ronde (2013) discutimos la posibilidad de considerar a las superposiciones cuánticas en términos de un enfoque paraconsistente. Argumentamos que, si bien la mayoría de las interpretaciones de la mecánica cuántica intenta escapar a las contradicciones, existen varias razones que indican la importancia de considerar un enfoque de este tipo. Recientemente, Arenhart y Krause (2016) han presentado numerosos argumentos en contra del enfoque paraconsistente a las superposiciones cuánticas. En este trabajo intentaré responder a las preguntas y obstáculos presentados por ellos. Argumentaré, en primer lugar, que los obstáculos discutidos por Arenhart y Krause se encuentran basados en una visión específica que toma como fundamento una metafísica actualista -la cual implícitamente presupone a la lógica cuántica. En segundo lugar, que existen muchas interpretaciones que investigan la posibilidad de desarrollar un modo de existencia diferente al actual, para los cuales nuestro enfoque paraconsistente puede resultar un prospecto interesante. Finalmente, proveemos un conjunto de respuestas específico a los problemas propuestos por Arenhart y Krause con el objetivo de clarificar la perspectiva original introducida por nuestro enfoque.

Palabras clave: superposiciones cuánticas - paraconsistencia - problema de la medición

* Received: 22 May 2018. Accepted in revised version: 7 August 2018.

† CONICET/Universidad de Buenos Aires/Universidad Nacional Arturo Jauretche (UNAJ), Argentina/Vrije Universiteit Brussel, Belgium. To contact the author, please write to: cderonde@gmail.com.

* We want to thank Nahuel Sznajderhaus for a careful reading of an earlier draft of this paper. Also to Décio Krause, Jonas Arenhart and Newton da Costa for many discussions on the subject. This work was partially supported by the following grants: FWO project G.0405.08 and FWO-research community W0.030.06. CONICET RES. 4541-12 (2013-2014) and PIO-CONICET-UNAJ (15520150100008CO) "Quantum Superpositions in Quantum Information Processing".

Metatheoria 9(2)(2019): 45-59. ISSN 1853-2322. eISSN 1853-2330.

(c) Editorial de la Universidad Nacional de Tres de Febrero. Publicado en la República Argentina. 


\section{Metaphysical stances and problems}

In da Costa and de Ronde (2013) we argued in favor of the possibility of considering quantum superpositions in terms of a paraconsistent approach. Even though most interpretations of Quantum Mechanics (QM) attempt to escape the debate about contradictions within superposed states, we presented several reasons why it could be worthwhile to engage in a research of this kind. In short, the PAQS calls the attention to the presence of contradictory statements within superposed "Schrödinger cat states"; e.g. 'the electron has spin up' and 'the electron has spin down', 'the atom is decayed' and 'the atom is not decayed', etc. Orthodox quantum logic escapes this debate by interpreting these statements in relation to their possible future actualization. However, such a reply leaves unanswered the question regarding the reference of such states in the present tense.

Recently, Arenhart and Krause (2016) have raised several arguments against the Paraconsistent Approach to Quantum Superpositions (PAQS). Their obstacles are condensed in the following six main statements:

I. The PAQS does not allow for contradictions due to its incompatibility with the Semantic Requirement, the Minimal Property Ascription Condition and the Paraconsistent Property Ascription.

II. The PAQS obscures the meaning of probability in QM.

III. The PAQS does not explain the measurement problem.

IV. Contrary to what is claimed by PAQS, contradictions are not observed in QM.

V. The PAQS inflates unnecessarily the population of the world with contradictions.

VI. The PAQS does not explain the vanishing of terms in the superposition after measurement.

First of all we must remark that in da Costa and de Ronde (2013) we did not propose an interpretation nor a metaphysical scheme but only called the attention to the need of considering all terms in a quantum superposition as being part of physical reality-not necessarily in the actual realm-and the possibility of doing so in formal terms through paraconsistent logics. However, including contradictions might be regarded as preparing the stage for a metaphysical step. This is why we believe that-now entering the scenery of metaphysics-the remarks and considerations of Arenhart and Krause deserve careful attention as well as answers. In this paper we will argue that:

i) Arenhart and Krause place their obstacles from a specific actualist metaphysical frame, which we will characterize in terms of what we call orthodoxy.

ii) That this is not necessarily the only possible representation of quantum physical reality. And that from the perspective of many interpretations which consider a realm of existence different to actuality, the PAQS can be regarded as a valuable prospect. Furthermore, that within such non-actualist metaphysical schemes, the problems and obstacles raised by Arenhart and Krause disappear.

In The Empirical Stance, Bas van Fraassen makes a remarkable claim: "The problem of appearance and reality affects first of all philosophy itself. I argue for a view of philosophy as a stance, as existential" (2002, p. xviii). To consider philosophy as a stance, as existential, means that our analysis is not void of values, intentions and presuppositions. These must be made explicit in order to be honest about the limits of our own problems and arguments. From this perspective-which we strongly support-, quite immediately we are driven into the specific consideration of the metaphysical presuppositions that we accept or not within a particular analysis. Physics does not escape this state of affairs. As remarked by Einstein: "The problem is that physics is a kind of metaphysics; physics describes 'reality'. But we do 
not know what 'reality' is. We know it only through physical description" (Curd \& Cover 1998, p. 1196). Indeed, due to the fact a physical problem is always constituted through a metaphysical perspective there is no neutral nor distant questioning regarding a physical problem. Posing a problem involves definite metaphysical presuppositions and choices without which questions remain meaningless. For example, the problem of interpreting QM, which makes complete sense from a realist perspective, is completely meaningless from an instrumentalist one which can understand the theory merely as an algorithm that predicts 'clicks' and is not related to physical reality. Instrumentalism has a set of problems different from those of realism. Furthermore, a problem determines-implicitly-its own set of possible answers, placing in itself a limit to knowledge and understanding. And it is through this same limit that we learn about the world. As we shall see, posing problems to QM is a subtle task which is sometimes betrayed by hidden agendas.

One might characterize the orthodox line of research in present philosophy of QM in direct relation to the metaphysics of actuality; a worldview which goes back to Newton's metaphysics of classical mechanics. If one considers the core of the classical (Newtonian) physical and metaphysical representation of the world ${ }^{1}$ one is then immediately stuck with two main concepts: the notion of 'entity' or 'object' and the notion of 'actuality'-understood not as hic et nunc observation, but rather as characterizing a mode of existence. Both notions were logically and metaphysically developed by Aristotle through a systematic conceptual architectonic. However, within Aristotle's hylemorphic scheme, also 'potentiality' played a major role. Movement was described in terms of the path from potentiality, which contained the undetermined, contradictory and non-individual realm of existence, to the actual mode of being, determined through the logical and ontological Principle of Existence (PE), the Principle of Non-Contradiction (PNC) and the Principle of Identity (PI). Thus the foundation of classical Aristotelian logic was concomitant with the foundation of his metaphysics (see Verelst \& Coecke 1999). Metaphysics is understood here as the systematic development of conceptual networks capable of providing representations of reality. In this sense, metaphysics is understood as a pre-requisite for discussing about physics.

The notion of entity was capable of unifying, of totalizing in terms of a "sameness", creating certain stability for knowledge to be possible. The transition from medieval to modern science coincides with the abolition of Aristotelian hylemorphic metaphysical scheme as the foundation of knowledge. However, the basic structure of Aristotelian logic still remained the basis for correct reasoning (Verelst \& Coecke 1999, p. 7). As a consequence, potentiality was completely eliminated from physics. After Newton, only actuality was considered in order to account for the mode of existence of physical objects. Above many different metaphysical schemes Greek Atomism became the triumphant metaphysical representation of nature. Within Newtonian mechanics every physical system (every atom) may be described exclusively by means of its actual properties, and its evolution, through their evolution. Thus, potential or possible properties are considered only as the points to which the system might arrive in a future instant of time. The physics of Newton became a physics of pure actuality, providing a description of the universe in terms of an actual state of affairs (i.e., sets of systems constituted by actual (definite valued) preexistent properties) evolving in time. ${ }^{2}$ It is in fact this particular metaphysical picture which has become the "common sense" of our time.

Going back to QM, the orthodox line of research in philosophy of QM has been based on this same actualist classical metaphysical scheme and seeks for answers in terms of the set of (metaphysical) presuppositions derived from it. Still today it is claimed-in a somewhat metaphorical sense-that "QM talks about elementary particles". However, from a methodological perspective it might seem not very smart to take as a standpoint, in order to solve problems, presuppositions which-we already know-

\footnotetext{
1 See for discussion de Ronde, Freytes \& Domenech (2014a, b).

2 As remarked by Dieks: "In classical physics the most fundamental description of a physical system (a point in phase space) reflects only the actual, and nothing that is merely possible. It is true that sometimes states involving probabilities occur in classical physics: think of the probability distributions $\rho$ in statistical mechanics. But the occurrence of possibilities in such cases merely reflects our ignorance about what is actual. The statistical states do not correspond to features of the actual system (unlike the case of the quantum mechanical superpositions), but quantify our lack of knowledge of those actual features" (2010, p. 124). See also for a detailed analysis de Ronde, Freytes \& Domenech (2014a).
} 
have problems with the theory in the first place. If everything seems to point in the direction that QM has problems with the notions of 'individuality', 'definiteness', 'space', 'time', 'locality', 'separability', etc. Why should we keep trying to solve problems assuming these very same concepts which are problematic for the theory? And why should we accept that the only way of posing problems is according to such classical metaphysical picture? We believe the answer to these questions is related to the importance of Bohr's philosophy of QM. Indeed, Bohr stressed repeatedly the idea that: "the unambiguous interpretation of any measurement must be essentially framed in terms of classical physical theories, and we may say that in this sense the language of Newton and Maxwell will remain the language of physicists for all time" (apud Wheeler \& Zurek 1983, p. 7). Closing the possibility of creating new physical concepts, he argued that "it would be a misconception to believe that the difficulties of the atomic theory may be evaded by eventually replacing the concepts of classical physics by new conceptual forms" (apud Wheeler \& Zurek 1983, p. 7). However, as remarked already by Dieks: "the demand that there should be a [classical] visualizable model would be tantamount to demand that classical physics should determine the conceptual tools of new theories. This would deny the possibility of really new fundamental theories, conceptually independent of classical physics" (1989, p. 1417). Due to the closure of the orthodox scheme, the problems have focused in trying to justify the classical description of the world in terms of entities constituted by properties in the actual mode of existence. ${ }^{3}$

In this respect, regarding the interpretation of QM, we might distinguish between two main strategies. The first strategy consists in beginning with a presupposed set of classical metaphysical principles and advance towards a new formalism that is able to account for such principles. Examples of this strategy are the collapse theory proposed by Ghirardi, Rimini and Weber (1986) which introduces non-linear terms in the Schrödinger equation in order to justify the collapse of the superposed state to one of its terms and Bohmian mechanics which introduces space-time particles (Bohm 1953) in order to escape the two evolutions present in the theory, one deterministic and the other indeterministic. The second strategy consists in accepting the orthodox formalism of QM and advance towards the creation and elucidation of the metaphysical principles and physical concepts which would allow us to answer the question: what is QM talking about? From this perspective, the importance is to focus on the formalism of the theory and try to learn about the symmetries, the logical features and structural relations. The idea is that, by learning about such aspects of the theory, we could also come up with the metaphysical conditions that should be developed in order to provide a coherent interpretation of QM. The second strategy opens the possibility of developing a different (non-actual) realm of existence which is not based on "common sense" actualism. In fact, there is a vast literature which investigates such an idea in the context of QM. Good examples are Heisenberg's interpretation in terms of the Aristotelian notion of potentia, Piron and Aerts operational potentialities, Popper's propensities, Margenau's latencies or the more recent work by Suarez, Dorato and Esfeld on dispositions and causal powers. ${ }^{4}$

\section{The superposition problem: beyond the metaphysics of actuality}

One of the main features of QM which seems to force the abandonment of our classical actualist understanding of physical reality is the existence of the so called "quantum superpositions". Quantum superpositions are mathematical elements (linear combinations of, in general, multiple states) which might relate to seemingly contradictory properties such as 'spin up' and 'spin down', 'decayed' and 'not decayed' or, as most dramatically exposed by Erwin Schrödinger (1935), even 'dead' and 'alive'. The projection operators we find in quantum superpositions give place to meaningful (probabilistic)

\footnotetext{
${ }^{3}$ These two Bohrian dictums, properly mixed with a realist flavor have created a set of "no-problems" about QM which have been intensively discussed for many decades: no-separability, no-individuality, no-locality, no-distributivity, no-identity, etc. These problems impose implicitly "right from the start" a set of classical (meta-physical) presuppositions within QM, namely, separability, individuality, locality, distributivity, identity, etc. All of them are mainly (metaphysical) problems which attempt to discuss and analyze the reference of the quantum formalism with respect to (classical) physical reality.

${ }^{4}$ See for a general review of these interpretations de Ronde (2011, Chap. 14) and references therein.
} 
physical statements which have been experimentally confirmed in accordance with the predictions of QM. Unfortunately, instead of advancing in the understanding of quantum superpositions, orthodoxy has concentrated on the famous Measurement Problem (MP), which is one of the main questions imposed by the "common sense" actualist metaphysics.

Measurement Problem (MP): Given a specific basis QM describes mathematically a state in terms of a superposition (of states). Since the evolution described by QM allow us to predict that the quantum system will get entangled with the apparatus and thus its pointer positions will also become a superposition, the question is why do we observe a single outcome instead of a superposition of them?

Although the MP accepts the fact that there is something very weird about quantum superpositions, leaving aside their problematic physical meaning and representation, it focuses on the justification of the actualization process and the observable outcomes. Taking as a standpoint the single actual result it asks: how do we get from the quantum superposition to a single measurement outcome? Assuming an empiricist stance, the MP is thus an attempt to justify why, regardless of QM, we only observe actuality. The problem places the result in the origin, what needs to be justified is the already known answer. But, could it be we were asking the wrong question?

Many interpretations have attempted to escape the ruling of actuality and classical logic. Going in line with these interpretations we have proposed an inversion of the MP. This new problem turns upside-down the focus, concentrating on the physical meaning of quantum superpositions themselvesinstead of trying to justify what we already know (de Ronde 2011). We believe that the answer to this new problem might be the key to truly understand the latest technical and experimental developments done today in laboratories around the world. This problem and our proposal go in line with an understanding of physical theories in terms of mathematical formalisms and physical concepts. As remarked by Heisenberg:

The history of physics is not only a sequence of experimental discoveries and observations, followed by their mathematical description; it is also a history of concepts. For an understanding of the phenomena the first condition is the introduction of adequate concepts. Only with the help of correct concepts can we really know what has been observed. (Heisenberg 1973, p. 264)

From this perspective a new problem can be defined:

Superposition Problem (SP): Given a situation in which there is a quantum superposition of more than one term, $\sum c_{i}\left|\alpha_{i}\right\rangle$, and given the fact that each one of the terms relates trough the Born rule to a meaningful physical statement, ${ }^{5}$ the problem is how do we represent this mathematical expression through adequate physical concepts.

The logical and ontological PE, PNC and PI created by Aristotle allowed Newton to develop a metaphysical representation of the physical world as an actual state of affairs. QM seems to have many difficulties to be interpreted following this formal and conceptual scheme. As a matter of fact QM has deep problems with each of these three (classical) metaphysical principles. It has problems with the PE, due to the contextual character of the theory which precludes the global valuation of the properties of a quantum system (Kochen \& Specker 1967). It has problems with the PI, due to the strange status of identical particles (French \& Krause 2006). And it also seems to have problems with the PNC, due to the existence of superpositions (da Costa \& de Ronde 2013).

Following Heisenberg, in the creation of a theory, both a formal and a conceptual developments takes place. In this respect, the question of contradiction within quantum superpositions cannot be addressed exclusively in formal terms, it requires both a formal and a conceptual level of analysis. It is

\footnotetext{
5 In de Ronde (2016a) we have defined this notion in the following terms. Meaningful Physical Statements: If given a specific situation a theory is capable of predicting in terms of definite physical statements the outcomes of possible measurements, then such physical statements are meaningful to the theory and must be constitutive parts of the particular representation of physical reality that the theory provides. Measurement outcomes must be considered only as an exposure of the empirical adequacy (or not) of the theory.
} 
in this particular sense that we believe that paraconsistent logics can be of great help. Just in the same way Krause himself has argued in favor of considering non reflexive logics and has even developed quasi-set theory in order to discuss quantum systems which do not obey the PI; the PAQS opens the door to the possibility of considering "right from the start" physical notions which are not restricted by the logical PNC. Exactly the same argument presented by Krause in favor of his quasi-set theory, according to which one should develop new formalisms in order to consider "right from the start" the main features of QM (Krause 1992) can be used in order to defend the PAQS. In fact, the history of physics is full of developments directly linked to formal shifts. For example, non-Euclidean geometry was a key formal development which allowed not only to produce new notions of space and time, but also to create the theory of relativity itself. So just in the same way as quasi-set theory has proven to be an interesting proposal in order to think about the problem of quantum non-individuals, the introduction of paraconsistent logics might provide a valuable help to investigate the physical meaning of quantum superpositions.

\section{Superpositions, potentiality and contradictions in QM}

It is a well-known fact in the foundational literature that projection operators cannot be interpreted as actual properties. The quantum probabilities that relate to each projection operator via the Born rule cannot be considered as being epistemic in case we attempt to provide a realist interpretation of the formalism. ${ }^{6}$ In fact, there is a feature which points in the direction of a realist interpretation of quantum possibility: the possibilities described by a superposition can interact with other quantum possibilities! This is also known in the literature as the interference of probabilities. As Dieks explains:

[...] in quantum mechanics the possibilities contained in the superposition state may interfere with each other. There is nothing comparable in classical physics. In statistical mechanics the possibilities contained in $\rho$ evolve separately from each other and do not have any mutual influence. Only one of these possibilities corresponds to the actual situation. (Dieks 2010, pp. 124-125)

But if we accept that quantum superpositions need not be described in terms of actual properties, a different (potential) realm might be then considered to account for them. In such case, we must be careful about the way in which we define contradictions. Obviously, if superpositions are considered as existents in a potential (non-actual) realm, the potential contradictions we find in Schrödinger cat states cannot be defined in terms of truth valuations to the actual realm. In fact, the modal KochenSpecker theorem shows explicitly that a quantum wave function implies multiple incompatible valuations which can be interpreted as potential contradictions (de Ronde, Freytes \& Domenech 2014a). This result might allow us to claim that while contradictions exist in the potential realm, they might not be found in actuality (de Ronde 2015).

Another important consequence of the ontological perspective towards quantum possibilities relates to the need of reconsidering the binary existential characterization of properties in terms of an homomorphic relation to the binary Boolean elements $\{0,1\}$ (or truth tables). In de Ronde (2016b) we proposed to extend the notion of element of physical reality escaping the characterization of existence in terms of certitude (probability $=1$ ) and considering "right from the start" the quantum probabilistic measure in objective terms. This move implies the development of existence beyond the gates of certitude and the complementary need of characterizing a new intensive ontology which relates existence to a value which pertains to the interval $[0,1]$. Our ontology in terms of immanent power has such an intensive characterization through the specification of a potentia. In this respect, we could say that, unlike properties (that pertain to systems) either exist or do not exist (i.e., they are related either to 1 or 0 ), immanent powers have a more complex characterization which requires, apart from its

\footnotetext{
${ }^{6}$ As it is well-known in the foundational literature of QM, the quantum probability measure is non-Kolmogorovian and cannot be interpreted in terms of ignorance (Rédei 2012). There exists an epistemic interpretation of QM known as QBism which following the Bayesian approach interprets probability in terms of knowledge of agents. The price QBism is willing to pay is the denial of the relation between QM and physical reality. According to the QBist view, QM does not make reference to physical reality, it is rather an algorithm to compute clicks in detectors (Fuchs, Mermin \& Schack 2014).
} 
binary relation to existence, a number pertaining to the closed interval $[0,1]$ which specifies its (potential) existence in an intensive manner. It is through the introduction of an intensive mode of existence that we can understand quantum probability as describing an objective feature of QM-rather than epistemic ignorance about an actual state of affairs.

We remark that the development of a potential realm also truly independent of the actual realm imposes the requirement of breaking down the idea that 'effectuations' can be only 'actual effectuations'. Today, strange as it might seem, the need to represent quantum superpositions does not seem to come from philosophical analysis or debates, it comes from the lab. Experimental physicists and computer scientists are presently using quantum superpositions in order to produce the most outstanding technical and experimental developments of the last decades. The paradox is that even though we can use superpositions to teleport information or implement quantum computers, we still cannot find the conceptual representation that would allow us to unify all we have learnt about them. Indeed, there are many characteristics and behaviors we know about superpositions: we know about their existence regardless of the effectuation of one of its terms, as shown, for example, by the interference of different possibilities in welcher-weg type experiments (Ma et. al. 2012), their reference to contradictory properties, as in Schrödinger cat states (Ourjoumtsev 2007), we also know about their non-standard route to actuality, as explicitly shown by the modal Kochen-Specker theorem (de Ronde, Freytes \& Domenech 2014a), and we even know about their non-classical interference with themselves and with other superpositions, used today within the latest technical developments in quantum information processing (Bernier et. al. 2013). As strange as it might seem, quantum computers use the fact that superposed quantum-bits can follow mutually (classically) incompatible paths simultaneously (Coecke, Heunen \& Kissinger 2013). This has led some to imagine (Wallace 2012) that quantum computers actually compute information in a multiverse of branching (classical) worlds!

It should be clear that the importance to find out how to physically represent quantum superpositions is not only philosophical but also technological, for it is only through adequate physical concepts that really new experiments could be imagined and designed. If we accept that quantum superpositions must be represented physically, then we should take into account that quantum possibilities interact and evolve according to the Schrödinger equation. It is also well known that one can produce interactions between multiple superpositions (entanglement) and then calculate the evolution of all terms, as well as predict the ratio of all probable outcomes. From a perspective which would attempt to provide a physical representation of superpositions, it then becomes difficult not to believe that these terms that 'interact', 'evolve' and 'can be predicted' according to the theory, are not (in some way) real-even though not actual. The consideration of these terms as real but not actual opens the possibility of considering potential contradictions which must be carefully defined in relation to their mode of existence in the potential realm and also in relation to the actual one. This project is a "parallel" and "entangled" project which must consider both formal and conceptual developments simultaneously.

\section{Answers to Arenhart and Krause}

Now that we have specified our standpoint, as well as the metaphysical stance implicitly assumed by Arenhart and Krause, we are ready to address more specifically some of the arguments and obstacles raised by them against the PAQS.

I. The PAQS does not allow for contradictions due to its incompatibility with the Semantic Requirement (SR), the Minimal Property Ascription Condition (MPAC) and the Paraconsistent Property Ascription (PPA).

\footnotetext{
${ }^{7}$ Even this multiplication of multiple (paraconsistent?) worlds could be addressed and discussed from the perspective presented by the PAQS.
} 
In order to criticize the notion of contradiction supposedly assumed by the PAQS, Arenhart and Krause (2016) present several conditions which, they claim, become incompatible within the approach. Firstly they propose the SR: "Contradictory statements [of the language] must have opposite truth values". To analyze a specific situation in QM they take the following quantum state: $\alpha\left|\uparrow_{x}\right\rangle+\beta\left|\downarrow_{x}\right\rangle$ and claim that: "The statements corresponding to properties represented by $\left|\uparrow_{\mathrm{x}}\right\rangle\left\langle\uparrow_{\mathrm{x}}\right|$ and $\left|\downarrow_{x}\right\rangle\left\langle\downarrow_{x}\right|$ must have opposite truth values" (p. 2). They then proceed to consider two property ascriptions, the MPAC: "If a system is in an eigenstate of an operator with eigenvalue $\mathrm{v}$, then the system has the qualitative property corresponding to such value of the observable." And the PPA: "When in a superposition, the system does have the properties related to the vectors forming the superposition, and they are contradictory" (p. 2). Arenhart and Krause then argue that:

[...] when the conditions for application of the minimal principle [MPAC] are met, both states have opposite truth values. But the job is still not done: we must still grant that one of those propositions must always be the case (being so that the other one will be false), as the semantic requirement [SR] for a contradiction seems to demand. (Arenhart \& Krause 2016, p. 5)

Their conclusion is then the following:

[...] it seems that the semantic requirement [SR] that one of the two terms in a superposition must always be the case (so that we can have a contradiction) is in fact in conflict with the paraconsistent property attribution [PPA] principle. For the latter principle to apply, in the case of a superposition, both 'up' and 'down' would have to be the case simultaneously. Recall what happens in the case of the two slit or Schrödinger's cat: according to [the PAQS] proposal, the particle must go by both slits, the cat must be dead and alive. So, there cannot be alternate truth values in this case, for both must be simply true. So, there is a conflict of the paraconsistent property attribution [PPA] principle with the very requirement that the vectors in a superposition stand for contradictory properties, at least according to the usual semantic requirements [SR] related to contradictions, as it appears in the traditional analysis of this concept. It seems that one cannot have both the claim that $u_{x}[|\uparrow x\rangle\langle\uparrow x|]$ and $d_{x}\left[\left|\downarrow_{x}\right\rangle\langle\downarrow x|\right]$ are contradictory and the claim that a superposition involves contradictions, as supplied by the paraconsistent property attribution [PPA] principle. As it stands, it seems, these demands are incompatible. (Arenhart \& Krause 2016, p. 5)

Let us analyze the presuppositions for the argument to stand. Both conditions, SR and MPAC, imply an analysis either in terms of actuality or actualization. But as we mentioned above, the notion of potential contradiction that we mean to put forward must consider a different potential realm truly independent of actuality. If the superposition is related to a potential realm of reality, such conditions cannot be taken into account for they implicitly assume that what is found out in actuality must be directly referred to the superposition irrespectively of its mode of existence. And we already know that both terms in a quantum superposition of the type $c_{1 x}\left|\uparrow_{\mathrm{x}}\right\rangle+c_{2 x}\left|\downarrow_{\mathrm{x}}\right\rangle$ cannot be considered as being actual. They are not actual contradictions, simply because the mode of existence of actuality-by definition-does not support contradictions. Or in other words, the notion of actual contradiction is simply an oximoron. However, if a different mode of existence is considered, then there is plenty of room to take these conditions into account within the PAQS. Let us see this in some more detail.

The existence of powers or capacities in Nature is a well-known subject of debate in meta-physics and philosophy of physics (Cartwright 1989, de Ronde2015, Dorato \& Esfeld 2010, Marmodoro 2010). An analogy with the measurement of an ontological power can show us why the argument of Krause and Arenhart does not follow irrespectively of the metaphysical considerations of the subject under study. Let us, for the sake of the argument, admit that immanent powers (which are not entities) exist in Nature, in a potential realm. ${ }^{8}$ An immanent power is one which relates to actuality through an immanent cause. This cause allows the power to exist independently of its actual effectuation. The immanent cause allows for the expression of effects remaining both in the effects and its cause. It does not only remain in itself in order to produce, but also, that which it produces stays within. Thus, in its

\footnotetext{
${ }^{8}$ For a detailed analysis of such interpretation in terms of immanent powers we refer to de Ronde $(2015,2016 b, 2017 \mathrm{a}, \mathrm{b})$.
} 
production of actual effects the potential does not deteriorate by becoming actual-as in the case of the hylemorphic scheme and causal powers. ${ }^{9}$

We can think, for example, of the immanent power, possessed by Messi, of shooting penal- ties. The power of shooting a penalty comprises contradictory actual effectuations of 'scoring a goal from the penalty' and also to 'fail the penalty' (following the PPA). These are potential contradictions. Let us see how this works. If Messi 'scores a goal' (in actuality) then everyone who is looking in the stadium will learn that Messi can score penalties, this means he possesses such a power (as demanded by the MPAC); and at the same time, everyone will have observed that (in actuality) he did not 'fail the penalty' (only one of the two possibilities will be 'true' in the actual realm as required by the SR)-and vice versa, when Messi 'fails the penalty'. ${ }^{10}$ The expression of a power in actuality exposes only partially its existence. But this is in no way different to the fact we can only observe the always partial perceptual adumbration of an object only when light shines upon it.

Does the measurement of an immanent power involve a collapse? The answer is no: the actual effectuation of the immanent power does not mean that the power has been destroyed nor that other powers have ceased to exist. ${ }^{11}$ The fact that Messi can shoot a penalty in actuality does not imply in any way that he ceases to have this power while he is not shooting penalties. Do we need actuality to claim that a power exists? The answer is no: Messi can choose not to shoot the penalty but nonetheless we can still claim that the power exists, even though we don't see it in actuality. Exactly in the same way, when I close my eyes, I can still claim that the table in front of me exists. This simple example attempts to show that if one moves away from the metaphysics of 'entities' and 'properties' in the actual realm, there might exist different ways to think about physical existence and actualization. The orthodox physical representation in terms of entities and properties in the actual mode of existence might not be the end of the road.

What is at stake in QM is the meaning itself of physical existence and reality. This is why we are not committed necessarily to a definite metaphysical stance, such as the one implied by orthodoxy. SR and MPAC are not necessary conditions for every interpretation of QM that we can think of. As presented by Arenhart and Krause these conditions are considered implicitly from a metaphysical stance in which reality is conceived only in terms of entities in the actual mode of existence. But, as we have shown above, if we include a different mode of existence within the conditions themselves, we can certainly take into account SR, MPAC and PPA within the PAQS. By claiming that PPA refers to the potential realm while MPCA refers to actualization (something we have discussed in detail in de Ronde, Freytes \& Domenech 2014a) and SR to the actual realm, all conditions are met by the PAQS and the problems raised by Arenhart and Krause disappear.

\section{The PAQS obscures the meaning of probability in QM.}

In several passages (e.g., pp. 4 and 5) Arenhart and Krause (2016) claim that PAQS does not allow for a good interpretation of probability in the context of QM. More specifically as they argue on p. 13:

Besides that lack of additional explanatory power or enlightenment on the theory [by the PAQS], there are some additional difficulties here. There is a complete lack of symmetry with the standard case of property attribution in quantum mechanics. As it is usually understood, by adopting the minimal property attribution principle, it is not contentious that when a system is in one eigenstate of an observable, then we may reasonably infer that the system has the property represented by the associated observable, so that the probability of obtaining the eigenvalue associated is 1 . In the case of superpositions, if they represented properties of their own, there is a complete disanalogy with that situation: probabilities play a different role, a system has a contradictory property attributed by a superposition irrespective of probability attribution and the role of probabilities in determining measurement outcomes. In a superposition, according to the proposal we are analyzing, probabilities play no role, the system simply has a given contradictory property by the simple fact of being in a (certain) superposition. (Arenhart \& Krause 2016, p. 13)

\footnotetext{
9 For a more detailed discussion of the notion of immanent cause we refer to de Ronde (2017b) and Melamed (2013, Chap. 2).

${ }^{10}$ This is an analogous situation to the one expressed in a situation in which we have the superposition $c_{1 x}\left|\uparrow_{x}\right\rangle+c_{2 x}\left|\downarrow_{x}\right\rangle$.

${ }^{11}$ See for a detailed discussion and analysis of immanent powers de Ronde (2017a, b).
} 
Some remarks go in order. Firstly, QM does not only talk about probabilities. The Kochen-Specker theorem, which we have mentioned before does not talk about probabilities but about actual definite values of observables. As a matter of fact, if QM would be only talking about probability, then there would be no single interpretational problem since the average values of all observables (commuting or not) are perfectly well defined in the theory according to the Born rule. The problem is that QM does not describe a mere ensemble of individuals. In fact, we do not even know what is an individual according to QM (see for an extensive discussion French \& Krasue 2006), neither is it clear what is the relation between preexistence and observation. As we know, due to its contextual character, it makes no sense to assume that a measurement exposes an already preexistent actual state of affairs. This is why MPAC needs to be so weak in the first place. Secondly, we do not know what it means that a superposition exists nor we understand how to relate it to actual observations. But claiming that the PAQS does not allow for a good interpretation of probability obviously would imply that we already know what quantum probability is talking about. This is simply not true. We do not know what quantum probability means in physical terms-beyond the mere instrumentalist reference to measurement outcomes. We do have a physical interpretation for classical (Kolmogorovian) probability, but we have no coherent interpretation of quantum (non-Kolmogorovian) probability. There is a whole literature regarding this point (Rédei 2012). In this respect, we can neither forget that the interpretations put forward by Popper, with his propensity interpretation of probabilities (Popper 1982), and by Bohm, with his causal interpretation; were specifically designed in order to find an answer to the meaning of quantum probability itself. The phrase "QM is a probabilistic theory", commonly used within the literature (and addressed by Arenhart \& Krause), is from our perspective: either an obvious mathematical statement with no interest since it only states the fact that in QM there is a (non-Kolmogorovian) probability measure assigned via Gleason's theorem, or a meaningless physical statement since we do not know what quantum probability is in conceptual terms. This was a fact already known to the founding fathers of the theory. As Schrödinger made the point in a letter to Einstein:

It seems to me that the concept of [physical] probability is terribly mishandled these days. [Physical] probability surely has as its substance a statement as to whether something is or is not the case -an uncertain statement, to be sure. But nevertheless it has meaning only if one is indeed convinced that the something in question quite definitely is or is not the case. A [physical] probabilistic assertion presupposes the full reality of its subject. (Apud Bub 1997, p. 115)

The problem with probability in QM is that due to the formalism there are serious inconveniences to assert the full reality of the subject in terms of an actual state of affairs. Apart from these remarks, in de Ronde (2016a) we have developed an interpretation of quantum probability consistent with potential contradictions and immanent powers.

Finally, we should also remark that Arenhart and Krause mix statements regarding the probability of obtaining one of the terms in a superposition, before and after the measurement has taken place (see e.g. pp. 4 and 10). As we shall argue in IV, one needs to be very careful regarding such analysis for it is also well known that conditional probability does not entail a necessary interpretation in terms of a collapse. ${ }^{12}$

\section{The PAQS does not explain the MP.}

Analyzing the PAQS, Arenhart and Krause also claim the following: "PAQS makes it even more difficult to understand how a typical measurement of a system in superposition yields always determinate results, but not contradictory results: one must be able to explain how a property possessed by the system disappears, while the other one remains" $(2016, \mathrm{p}$. 6). This remark is focused on the MP and the explanation of the phenomenon in terms of systems with properties in the actual

\footnotetext{
${ }^{12}$ As a matter of fact, the conditional probability of obtaining $B$ at $t_{2}$ given that $A$ was observed at $t 1, p\left(B, t_{2} \mid A, t_{1}\right)$, does not relate in any way the probabilities of measuring $A$ at $t_{1}, p\left(A, t_{1}\right)$, and the probability of measuring $B$ at $t_{2}, p\left(B, t_{2}\right)$. I am indebted to Prof. Dieks for pointing out to me this subtle point of non-collapse interpretations. See for discussion Dickson (1998).
} 
realm of existence. However, as we have argued above, once we consider two different realms of existence, the question of effectuation cannot be posed exclusively in terms of actuality.

Arenhart and Krause claim that the terms which do not get actualized after measurement suddenly "disappear". But this is an interpretational stance with respect to the Projection Postulate which is not self-evident and presupposes the idea of a "collapse"; i.e., a physical process that is not described by Schrödinger's evolution. Our previous example provides an intuitive non-collapse interpretation of what is going on. The immanent power of Messi to score a penalty exists independently of its particular actual effectuation. What happens in actuality does not transform the power. Thus, when Messi actually shoots the penalty-due to the immanent cause-the power does not disappear. Messi can shoot as many penalties as he wishes or he can also choose not to shoot any penalty. Whatever he does in the actual realm his power will not cease to exist (in the potential realm of existence). We will come back to this interpretational maneuver in VI. ${ }^{13}$

\section{Contrary to what is claimed by PAQS, contradictions are not observed in QM.}

From a realist perspective, observation cannot be regarded as a "self-evident given". As Einstein told to Heisenberg long time ago, from a realist perspective, "it is only the theory which decides what can be observed." In fact, the meaning of observation has remained a subtle point of debate, one at the heart of many interpretational problems of quantum theory. Arenhart and Krause have claimed-focusing once again on the MP-that: "For another disanalogy [of PAQS] with the usual case, one does not expect to observe a system in such a contradictory state: every measurement gives us a system in a particular state, never in a superposition." (da Costa $\&$ de Ronde, p. 10) But this is also true for physical objects with respect to identities. One does not observe identities either. Contradictions and identities are not observable, they are-on the contrary-the conditions of possibility which allow us to make sense of phenomena. As Hume had clearly exposed in his analysis of causation, our categorical understanding is not something "we find outside in the world" but, as Kant would later explain, something that we subjects impose in order to shape and configure our own experience. We do not see identities walking in the empirical world, neither have we observed the principle of non-contradiction.

Furthermore, we have already criticized the idea that observation in QM must be necessarily considered in terms of classical space-time experience (de Ronde 2014). When we say that according to Newtonian mechanics a cup falls to the floor accelerated at $9.8 \mathrm{~m}$ in $t_{1}$ seconds, there is a coherency of the statement, of the concepts implicitly used (object, space, time, etc.), the formal prediction (according to the equation of motion) and experience itself. But in QM, because we do not have a coherent language that makes contact with the formal structure, experience is not really well defined. Instead, what we have is a weird discourse which constantly contradicts itself. When we talk about "quantum particles", we know in fact that QM does not really talk about "particles". Some people argue that "this is just a way of talking". As we have attempted to show in this paper, language and, more specifically, physical concepts determine a definite perspective regarding problems and their solutions articulating our possibilities to think about experience.

One cannot simply "observe contradictions". 'Contradictions', like 'identity' or 'causality', are not something that we "find outside in the world". They are instead the basic metaphysical presuppositions that shape our theories in order to comprise and make sense of experience. The fact that contradictions are observed, or not, needs to be addressed from the standpoint of a coherent interpretation of QM, something we still do not have. What we should recognize is that at the present stage of our knowledge, we do not even know what is the cause of a 'click' in an EPR type experiment (de Ronde 2014, pp. 104-105).

\section{The PAQS inflates unnecessarily the population of the world with contradictions.} According to Arenhart and Krause:

\footnotetext{
${ }^{13}$ For a more detailed analysis of the quantum measurement process see de Ronde (2017a)
} 
[...] when one takes into account other virtues of a metaphysical theory, such as economy and simplicity, the paraconsistent approach seems to inflate too much the population of our world. In the presence of more economical candidates doing the same job and absence of other grounds on which to choose the competing proposals, the more economical approaches take advantage. Furthermore, considering economy and the existence of theories not postulating contradictions in quantum mechanics, it seems reasonable to employ Priest's razor-the principle according to which one should not assume contradictions beyond necessity (see Priest 1987)-and stick with the consistent approaches. Once again, a useful methodological principle seems to deem the interpretation of superposition as contradiction as unnecessary. (Arenhart \& Krause 2016, p. 10)

On the one hand, Arenhart and Krause claim that there are more economical interpretations which do exactly the same job the PAQS does. But what is the job done by the PAQS? The PAQS brings forward the possibility to interpret all terms in the superposition as physically existent; supporting the fact that all terms can be 'described', 'put to interact' and 'be predicted' through the Born rule and the Schrödinger equation of motion. The price that the interpretations that follow the PAQS might need to pay is giving up the metaphysical equation: Actuality = Reality. Thus, the PAQS allow us to investigate, in formal terms, the possibility of considering a contradictory (potential) realm independent of actuality. The PAQS does the job of developing formally a realm in which all terms of the superposition are allowed to exist, regardless of actuality. ${ }^{14}$ In the sense just discussed, and contrary to the claim put forward by Arenhart and Krause, the PAQS opens possibilities of development which have not yet been fully investigated.

It should be also clear that we are not claiming that all terms in the superposition are actual-as in the many worlds interpretations-overpopulating existence with unobservable actualities. What we claim is that PAQS opens the door to consider all terms as existent within a potential realmindependently of actuality. We claim that just like we need all properties to characterize a physical object, all terms in the superposition are needed for a proper characterization of what exists according to QM. We do not believe that this is overpopulating metaphysically the world with contradictions, but rather an attempt to take into account what both the formalism of QM (in terms of its meaningful physical statements) as well as physical experience in the laboratory seems to be telling us.

Finally, it is important to remark that-as we discussed in IV-such contradictory potentialities are observable just in the same way as actual properties can be observed in an object. Potentialities can be observed through actual effectuations in analogous fashion to how physical objects are observed. We never observe all perspectives of a classical object simultaneously, we only observe-at most-the partial perceptual adumbration of an object (i.e., a subset of actual properties).

\section{The PAQS does not explain the vanishing of terms in the superposition after measurement.} Finally, according to Arenhart and Krause:

[...] a new problem is created by this interpretation [PAQS], because besides explaining what is it that makes a measurement give a specific result when the system measured is in a superposition (a problem usually addressed by the collapse postulate, which seems to be out of fashion now), one must also explain why and how the contradictory properties that do not get actualized vanish. That is, besides explaining how one particular property gets actual, one must explain how the properties posed by the system that did not get actual vanish. (Arenhart \& Krause 2016, pp. 10-11)

Arenhart and Krause seem to assume that once the populated world of contradictions is measured all terms except one suddenly disappear. This implies an interpretation of the Projection Postulate in terms of a "collapse" (i.e., a physical interaction) of the quantum wave function. But, it is well known that such collapse interpretation is not necessarily the only possible interpretation. We are inclined to assume a non-collapse interpretation of the Projection Postulate while still considering the specificity of the actualization process in QM (see for discussion de Ronde, Freytes \& Domenech 2014a).

\footnotetext{
${ }^{14}$ Although we believe there is plenty of room to use the PAQS in many interpretations of QM, the author of this paper has argued elsewhere in favor of a non-collapse interpretation which considers the potential realm completely independent of actuality. We will discuss the particular relation of this interpretation to PAQS in da Costa \& de Ronde (2015).
} 
We might also recall that this is an important point for modal interpretations. As Vermaas makes the point:

In modal interpretations the state is [...] not updated if a certain state of affairs becomes actual. The non-actualized possibilities are not removed from the description of a system and this state therefor codifies not only what is presently actual but also what was presently possible. These non-actualized possibilities can, as a consequence, in principle still affect the course of later events. (Vermaas 1999, p. 295)

In line with the non-collapse solution to the MP proposed by modal interpretations, PAQS might allow us to claim that the superpositions remain existent (in potentiality) independently of their measurement (in actuality).

\section{Final remarks}

Arenhart and Krause have also called the attention to the understanding of contradiction via the Square of Opposition. Elsewhere, together with Domenech and Freytes, we have also analyzed via the Square of Opposition the meaning of quantum possibility. We argued that the notion of possibility would need to be discussed in terms of the formal structure of the theory itself and that, in such case, one should not study the Classical Square of Opposition but rather an Orthomodular Square of Opposition such as the one explicitly developed in Freytes, de Ronde and Domenech (2012). In de Ronde, Freytes and Domenech (2014b) we provided an interpretation of the Orthomodular Square of Opposition in terms of the notion of potentiality. In a future work we plan to analyze the proposal of Arenhart and Krause and discuss the meaning of contradiction relating our Orthomodular Square of Opposition with the constraints implied by the modal Kochen-Specker theorem. We expect that this analysis will provide us with a better understanding of contradictions in QM.

As we have shown, the criticisms of Arenhart and Krause arise mainly by assuming the orthodox metaphysics of actuality to which we never committed ourselves. This metaphysical scheme precludes "right from the start" any paraconsistent development. In contradistinction, PAQS allows us to consider a contradictory (non-actual) realm in which all terms of the superpositions preexist to actual measurements. A conclusion which goes in line with our present understanding of the formalism and the impossibility to interpret superpositions in terms of ignorance. In turn, this may allow us to provide a physical interpretation in accordance to the latest technical and experimental developments that are taking place today (e.g., quantum computation, quantum teleportation, quantum information processing, etc.) which make use of the interaction of all terms in the superposition irrespectively of their actual effectuations. ${ }^{15}$ It is important to remark that some new paraconsistent formalizations of quantum superpositions-which go in line with the original proposal presented in da Costa and de Ronde (2013)-are also being further developed (da Costa \& de Ronde 2015, Eva 2016, Krause \& Arenhart 2015).

We believe that science is about confronting the unknown, it implies a humble attitude with respect to experience and a critical understanding of the presuppositions we are willing to make. Science implies the creation and production of new ways of understanding reality; it is not about trying to justify that which we already know. But exactly because science has always taken advantage from opposite views and perspectives, we believe that our approach and line of research, although still speculative and in early stages of development, deserves the chance of being further developed.

\footnotetext{
${ }^{15}$ In this respect, the PAQS goes in line, for example, with the ongoing research of the quantum group at Oxford University Computing Laboratory directed by Abramsky and Coecke and the projects about quantum interaction directed by Smets at Amsterdam University.
} 


\section{References}

Arenhart, J. R. and D. Krause (2016), “Contradiction, Quantum Mechanics, and the Square of Opposition”, Logique et Analyse 59: 273-281.

Bernien, H., Hensen, B., Pfaff, W., Koolstra, G., Blok, M. S., Robledo, L., Taminiau, T. H., Markham, M., Twitchen, D. J., Childress, L. and R. Hanson (2013), "Heralded Entanglement Between SolidSstate Qubits Separated by Three Metres", Nature 497: 86-90.

Bohm, D. (1953), "Proof That Probability Density Approaches $|\psi|^{2}$ in Causal Interpretation of the Quantum Theory", Physical Review 89: 458-466.

Bub, J. (1997), Interpreting the Quantum World, Cambridge: Cambridge University Press.

Cartwright, N. (1989), Nature's Capacities and Their Measurements, Oxford: Clarendon Press.

Coecke, B., Heunen, C. and A. Kissinger (2013), "Compositional Quantum Logic", in Coecke, B., Ong L. and P. Panangaden (eds.), Computation, Logic, Games, and Quantum Foundations, Singapore: Springer, pp. 21-36.

Curd, M. and J. A. Cover (1998), Philosophy of Science. The Central Issues, New York: W. W. Norton \& Company.

da Costa, N. and C. de Ronde (2013), "The Paraconsistent Logic of Quantum Superpositions", Foundations of Physics 43: 845-858.

da Costa, N. and C. de Ronde (2015), "The Paraconsistent Approach to Quantum Superpositions Reloaded: Formalizing Contradictory Powers in the Potential Realm”, preprint.

de Ronde, C. (2011), The Contextual and Modal Character of Quantum Mechanics: A Formal and Philosophical Analysis in the Foundations of Physics, PhD dissertation, Utrecht: Utrecht University.

de Ronde, C. (2014), "The Problem of Representation and Experience in Quantum Mechanics", in Aerts, D., Aerts S. and C. de Ronde (eds.), Probing the Meaning of Quantum Mechanics: Physical, Philosophical and Logical Perspectives, Singapore: World Scientific, pp. 91-111.

de Ronde, C. (2015), "Modality, Potentiality and Contradiction in Quantum Mechanics", in Beziau, J.-Y., Chakraborty, M. and S. Dutta (eds.), New Directions in Paraconsistent Logic, Berlin: Springer, pp. 249-265.

de Ronde, C. (2016a), "Quantum Superpositions and the Representation of Physical Reality Beyond Measurement Outcomes and Mathematical Structures", submitted (quant- ph/arXiv:1603.06112).

de Ronde, C. (2016b), "Probabilistic Knowledge as Objective Knowledge in Quantum Mechanics: Potential Powers Instead of Actual Properties", in Aerts, D., de Ronde, C., Freytes, H. and R. Giuntini (eds.), Probing the Meaning and Structure of Quantum Mechanics: Superpositions, Semantics, Dynamics and Identity, Singapore: World Scientific, pp. $141-178$.

de Ronde, C. (2017), "Causality and the Modeling of the Measurement Process in Quantum Theory", Disputatio, forthcoming.

de Ronde, C. (2017), "Immanent Powers versus Causal Powers (Propensities, Latencies and Dispositions) in Quantum Mechanics", in Aerts, D., Dalla Chiara, M. L., Krause, D. and C. de Ronde (eds.), Probing the Meaning of Quantum Mechanics, Singapore: World Scientific, forthcoming.

de Ronde, C., Freytes, H. and G. Domenech (2014a), "Interpreting the Modal Kochen-Specker Theorem: Possibility and Many Worlds in Quantum Mechanics", Studies in History and Philosophy of Modern Physics 45: 11-18.

de Ronde, C., Freytes, H. and G. Domenech (2014b), "Quantum Mechanics and the Interpretation of the Orthomodular Square of Opposition”, in Béziau, J.-Y. and K. Gan-Krzywoszynska (eds.), New Dimensions of the Square of Opposition, Munich: Philosophia Verlag, pp. 223-242.

de Ronde, C., Freytes, H. and G. Domenech (2014), "Quantum Logic in Historical and Philosophical Perspective", Internet Encyclopedia of Philosophy, URL = http://www.iep.utm.edu/qu-logic/.

Dickson, W. M. (1998), Quantum Chance and Nonlocality: Probability and Nonlocality in the Interpretations of Quantum Mechanics, Cambridge: Cambridge University Press. 
Dieks, D. (1989), “Quantum Mechanics Without the Projection Postulate and Its Realistic Interpretation”, Foundations of Physics 19: 1397-1423.

Dieks, D. (2010), “Quantum Mechanics, Chance and Modality”, Philosophica 83: 117-137.

Dorato, M. and M. Esfeld (2010), "GRW as an Ontology of Dispositions”, Studies in History and Philosophy of Modern Physics 41: 41-49.

Eva, B. (2016), “A Topos Theoretic Framework for Paraconsistent Quantum Theory”, in Aerts, D., de Ronde, C., Freytes, H. and R. Giuntini (eds.), Probing the Meaning of Quantum Mechanics: Superpositions, Dynamics, Semantics and Identity, Singapore: World Scientific, pp. 340-350.

French, S. and D. Krause (2006), Identity in Physics: A Historical, Philosophical and Formal Analysis, Oxford: Oxford University Press.

Freytes, H., de Ronde, C. and G. Domenech (2012), "The Square of Opposition in Orthod-Modular Logic", in Béziau, J.-Y. and D. Jacquette (eds.), Around and Beyond the Square of Opposition: Studies in Universal Logic, Basel: Springer, pp. 193-201.

Fuchs, C. A., Mermin, N. D. and R. Schack (2014), “An Introduction to QBism with An Application to the Locality of Quantum Mechanics", American Journal of Physics 82: 749. (quant-ph/arXiv:1311.5253)

Ghirardi, G. C., Rimini A. and T. Weber (1986), "Unified Dynamics for Microscopic and Macroscopic Systems", Physical Review D 34: 470-491.

Heisenberg, W. (1973), "Development of Concepts in the History of Quantum Theory", in Mehra, J. (ed.), The Physicist's Conception of Nature, Dordrecht: Reidel, pp. 264-275.

Krause, D. (1992), “On a Quasi-Set Theory”, Notre Dame Journal of Formal Logic 33: 402- 411.

Krause, D. and J. Arenhart (2015), "A Logical Account of Superpositions”, in Aerts, D., de Ronde, C., Freytes, H. and R. Giuntini (eds.), Probing the Meaning and Structure of Quantum Mechanics: Superpositions, Semantics, Dynamics and Identity, Singapore: World Scientific, pp. 44-59.

Kochen, S. and E. Specker (1967), "On the Problem of Hidden Variables in Quantum Mechanics", Journal of Mathematics and Mechanics 17: 59-87. (Reprinted in Hooker, C. A. (ed.), The Logico-Algebraic Approach to Quantum Mechanics, vol. I, Dordrecht: Reidel, 1975, pp. 293-328.)

Ma, X., Zotter, S., Kofler, J., Ursin, R., Jennewein, T., Brukner, C. and A. Zeilinger (2012), “Experimental DelayedChoice Entanglement Swapping", Nature Physics 8: 480-485.

Marmodoro, A. (ed.) (2010), The Metaphysics of Powers: Their Grounding and Their Manifestations, New York: Routledge.

Melamed, Y. (2013), Spinoza's Metaphysics and Thought, Oxford: Oxford University Press.

Ourjoumtsev, A., Jeong, H., Tualle-Brouri, R. and P. Grangier (2007), “Generation of Optical 'Schrödinger Cats' from Photon Number States", Nature 448: 784-786.

Popper, K. R. (1982), Quantum Theory and the Schism in Physics, New Jersey: Rowman and Littlefield.

Rédei, M. (2012), "Some Historical and Philosophical Aspects of Quantum Probability Theory and its Interpretation", in Dieks, D., Gonzalez, W. J., Hartmann, S., Stöltzner, M. and M. Weber (eds.), Probabilities, Laws, and Structures, Berlin: Springer, pp. 497-506.

Schrödinger, E. (1935), "Die gegenwärtige Situation in der Quantenmechanik”, Die Naturwissenschaften 23: 807-812, 823-828, 844-849. (English version: "The Present Situation in Quantum Mechanics”, in Wheeler, J. A. and W. H. Zurek (eds.), Quantum Theory and Measurement, 1983, Princeton: Princeton University Press, pp. 152-167.)

Van Fraassen, B. C. (2002), The Empirical Stance, New Haven: Yale University Press.

Verelst, K. and Coecke, B. (1999), "Early Greek Thought and perspectives for the Interpretation of Quantum Mechanics: Preliminaries to an Ontological Approach”, in Aerts, D. (ed.), The Blue Book of Einstein Meets Magritte, Dordrecht: Kluwer, pp. 163-196.

Vermaas, P. E. (1999), A Philosopher's Understanding of Quantum Mechanics, Cambridge: Cambridge University Press.

Wallace, D. (2012), The Emergent Multiverse: Quantum Theory according to the Everett Interpretation, Oxford: Oxford University Press.

Wheeler, J. A. and W. H. Zurek (eds.) (1983), Theory and Measurement, Princeton: Princeton University Press. 